\title{
羰基还原酶在动态动力学拆分中的应用进展
}

\author{
倪国伟 $a, b$ 汤佳伟 ${ }^{a}$ 邹 杰 ${ }^{a}$ 陈少欣 ${ }^{a}$ 鞠佃文*,b 张福利*, $a$ \\ $\left({ }^{a}\right.$ 中国医药工业研究总院 上海医药工业研究院 创新药物与制药工艺国家重点实验室 上海 201203) \\ $\left({ }^{b}\right.$ 复旦大学药学院 上海 201203)
}

\begin{abstract}
摘要 生物催化是不对称催化中制备手性药物的重要方法之一，具有化学、区域与高立体选择性的特点. 随着 DNA 测 序与合成技术以及蛋白工程技术的突破, 研究者可以快速获得基础与应用研究所需要的酶. 近年来, 生物催化在不对 称催化中的研究较为活跃. 在广泛应用的生物催化剂中, 羰基还原酶能够高立体选择性地还原羰基为单一手性中心的 仲醇. 当将羰基还原酶应用于动态动力学拆分时, 可经一步还原, 高立体选择性地制备含两个手性中心的仲醇. 综述 了其原理并梳理近十年的研究案例, 最后尝试提供一种研究思路: 篮酶-消旋一平衡, 以期为基础与应用研究提供思路. 关键词 不对称催化; 生物催化; 羊基还原酶; 动态动力学拆分
\end{abstract}

\section{Recent Advances on Carbonyl Reductases for Dynamic Kinetic Resolution}

\author{
Ni, Guowei ${ }^{a, b} \quad$ Tang, Jiawei $^{a} \quad$ Zou, Jie $^{a} \quad$ Chen, Shaoxin $^{a}$ \\ Ju, Dianwen ${ }^{*, b} \quad$ Zhang, Fuli ${ }^{*, a}$ \\ ( ${ }^{a}$ State Key Laboratory of New Drug and Pharmaceutical Process, Shanghai Institute of Pharmaceutical Industry, \\ China State Institute of Pharmaceutical Industry, Shanghai 201203) \\ ( ${ }^{b}$ School of Pharmacy, Fudan University, Shanghai 201203)
}

\begin{abstract}
Biocatalysis is a basic method of asymmetric catalysis for preparing chiral Active Pharmaceutical Ingredients, which owns several "green merits": chemo-, regio- and high enantioselectivity. As the development of DNA seqencing, DNA synthesis and protein engineering, suitable enzymes can be efficiently developed for basic researches and industrial applications. Biocatalysis has been keeping as a hot spot in asymmetric synthesis recently. Carbonyl reductases have been widely used for stereoselectively transforming ketones to chiral second alcohols with only one stereocenter. When combining with Dynamic Kinetic Resolution (DKR), the bioreaction with carbonyl reductases can efficiently construct chiral alcohols with two stereocenters in one step. This review highlights the method of its mechanism and nearly twenty examples from research papers and patents for one decade. We attempt to analyze and conclude the characteristics of this method based on chemical structures and enzymes. At last, a practical and developing research method is recommended in three steps: screening-racemization-balance in sequence. It is hoped to be useful for future basic researches and industrial applications.
\end{abstract}

Keywords asymmetric catalysis; biocatalysis; carbonyl reductases; dynamic kinetic resolution

不对称催化包括三种类型：金属配体催化、有机小 分子催化和生物催化. 生物催化又称为生物转化, 是指 利用酶或有机体(细胞、细胞器等)作为催化剂实现化学 转化的过程 ${ }^{[1]}$. 在多样的生物催化剂中, 水解酶、氧化还 原酶和转移酶应用较为广泛. 据统计, 水解酶使用占比 约 $60 \%$, 氧化还原酶类使用占比约 $20 \%{ }^{[2]}$. 羰基还原酶 (Carbonyl Reductase CRED), 又称酮还 (Ketoredutase KRED)或者醇脱氢酶(Alcohol Dehydrogenase, ADH), 属
于氧化还原酶类, 广泛存在于细菌、真菌、酵母和动植 物体内. 在辅酶 NADH 或 NADPH 条件下，羰基还原酶 能将酮或醛转化成醇类化合物 ${ }^{[3]}$. 由于辅酶价格昂贵, 研究人员成功开发了高效且低成本的辅酶再生方法, 以 减少辅酶用量. 工业上常利用醇脱氢酶/异丙醇 ${ }^{[4]}$ 、甲酸 脱氢酶/甲酸铵 ${ }^{[5]}$ 或葡萄糖脱氢酶/葡萄糖 ${ }^{[6]}$ 三种组合方 法实现辅酶 NAD $(\mathrm{P}) \mathrm{H}$ 的循环. 特别地, 对于醇脱氢酶/ 异丙醇体系, 部分醇脱氢酶具有双向催化特征: 一是催

* Corresponding authors. E-mail: zhangfuli1@sinopharm.com; dianwenju@fudan.edu.cn
Received June 7,2018; revised August 7,2018; published online September 10,2018. 
化还原羰基; 二是催化氧化异丙醇脱氢, 实现辅酶再 生 ${ }^{[7]}$, 单酶即可实现双酶的催化功能. 近些年, 利用整 细胞或分离酶催化该类还原反应已成功应用于一系列 工业转化过程中 ${ }^{[8 \sim 19]}$ (表 1 和图 1).

羰基还原酶是一种实用的能够实现不对称还原的 生物催化剂, 因其具有化学、区域与高立体选择性的特
点，正逐渐成为合成手性醇的主要方法之一 ${ }^{[20]}$. 羰基还 原酶应用于工业化生产的生物转化案例，其特征主要表 现为底物浓度、转化率、手性纯度、酶的用量和反应时 间等参数应达到表 2 所列的数值, 如底物浓度 $\geqslant 100$ $\mathrm{g} / \mathrm{L}$ ，手性纯度 $\geqslant 99 \%{ }^{[21]}$. 从生物催化剂成本考虑，由于 DNA 测序与合成技术的突破，生产酶的成本已经有大

表 1 羰基还原酶在重大药物品种的工业化应用

Table 1 Pharmaceutical drugs applied with carbonyl reductases in industry

\begin{tabular}{|c|c|c|c|}
\hline 通用名 & 美国上市年份 & 适应症 & 手性砌块 \\
\hline 阿托伐他汀 ${ }^{[8]}$ & 1996 & 高胆固醇血症 & \\
\hline 孟鲁司特 ${ }^{[9]}$ & 1998 & 哮喘 & \\
\hline 依折麦布 ${ }^{[10]}$ & 2002 & 高胆固醇血症 & \\
\hline 阿扎那韦 ${ }^{[11]}$ & 2003 & 艾滋病感染 & \\
\hline 阿瑞匹坦 ${ }^{[12]}$ & 2003 & 化疗止吐 & \\
\hline 度洛西汀 ${ }^{[13]}$ & 2004 & 重度抑郁症 & \\
\hline 索利那新 ${ }^{[14,15]}$ & 2004 & 膀胱过动症 & \\
\hline 替格瑞洛 ${ }^{[16,17]}$ & 2011 & 血栓中风 & \\
\hline 克唑替尼 ${ }^{[18]}$ & 2011 & 非小细胞肺癌 & \\
\hline 依鲁替尼 ${ }^{[19]}$ & 2013 & 慢性淋巴细胞白血病 & \\
\hline
\end{tabular}


<smiles>CC(C)c1c(C(=O)Nc2ccccc2)c(-c2ccccc2)c(-c2ccc(F)cc2)n1CC[C@H](O)C[C@H](O)CC(=O)O</smiles>

阿托伐他汀<smiles>CC(C)(O)c1ccccc1CC[C@H](SCC1(CC(=O)O)CC1)c1cccc(/C=C/c2ccc3ccc(Cl)cc3n2)c1</smiles>

孟鲁斯特<smiles>O=C1[C@H](CCC(O)c2ccc(F)cc2)[C@H](c2ccc(O)cc2)N1c1ccc(F)cc1</smiles>

依折麦布<smiles>C[C@@H](O[C@@H]1OCCN(Cc2n[nH]c(=O)[nH]2)[C@H]1c1ccc(F)cc1)c1cc(C(F)(F)F)cc(C(F)(F)F)c1</smiles>

阿瑞匹坦<smiles>CCNC[C@H](O)[C@H](Cc1ccccc1)NC(=O)[C@@H](NC(=O)OC)C(C)(C)C</smiles>

阿扎那韦<smiles>CCC(=O)NC(C(=O)NI)C(C)(C)C</smiles><smiles>CNCC[C@H](Oc1cccc2ccccc12)c1cccs1</smiles>

度洛西汀<smiles>O=C(O[C@@H]1CN2CCC1CC2)N1CCc2ccccc2[C@H]1c1ccccc1</smiles>

索利那新<smiles>CC(Oc1cc(-c2cnn(C3CCNCC3)c2)cnc1N)c1c(Cl)ccc(F)c1Cl</smiles><smiles>CCCSc1nc(N[C@H]2CC2c2ccc(F)c(F)c2)c2nnn(C3C[C@H](OCCO)[C@@H](O)C3O)c2n1</smiles>

替格瑞洛<smiles>C=CC(=O)N1CCC[C@H](n2nc(-c3ccc(Oc4ccccc4)cc3)c3c(N)ncnc32)C1</smiles>

依鲁替尼

图 1 药物的化学结构

Figure 1 Structures of pharmaceutical drugs

表 2 羰基还原酶的工业化标准

Table 2 Industrial standard for carbonyl reductase

\begin{tabular}{cc}
\hline 工艺参数 & 数值 \\
\hline 底物浓度 & $\geqslant 100 \mathrm{~g} / \mathrm{L}$ \\
转化率 & $\geqslant 98 \%$ \\
手性纯度 & $\geqslant 99 \%$ \\
反应时间 & $\leqslant 24 \mathrm{~h}$ \\
酶用量 & $\leqslant 5 \mathrm{~g} / \mathrm{L}$ \\
\hline
\end{tabular}

幅度的降低, 且随着生产规模的扩大, 酶的成本会进一

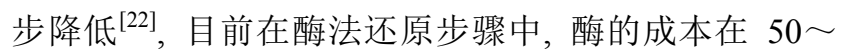
100 元/千克产品(如表 3). 随着新的羰基还原酶持续不 断的涌现, 为有机合成专家提供了丰富的手性催化剂工 具箱, 同时越来越多的合成药物学家在早期新药合成工 艺开发的阶段, 已经尝试将生物催化反应应用于路线的 设计中 ${ }^{[23,24]}$.

羰基还原酶在动态动力学拆分中应用的案例多散 落在不同的文献, 很少有研究者对其进行梳理与归纳, 本文以此为切入点对其进行综述. 为便于理解, 首先 介绍动力学拆分 $(\mathrm{KR})$ 与动态动力学拆分(DKR)的基本 概念. 以脂肪酶拆分仲醇制备单一异构体为例加以解释 动力学拆分 ${ }^{[25]}$. 动力学拆分是指在脂肪酶的催化下, 仲
表 3 不同生产规模下酶的成本比较

Table 3 Comparison of cost in different scales

\begin{tabular}{lcc}
\hline 工业规模 & 产品成本 $/\left(\right.$ 元・ $\left.\mathrm{kg}^{-1}\right)$ & 酶成本 $/\left(\right.$ 元 $\left.\bullet \mathrm{kg}^{-1}\right)$ \\
\hline 药物 & $>760$ & 76 \\
精细化工 & $>114$ & 11.4 \\
特殊化学品 & 38 & 1.9 \\
大宗产品 & 7.6 & 0.38 \\
\hline
\end{tabular}

醇中的一个对映体发生成酯反应，而另一个对映异构体 不参与或少量参与反应, 其理论收率为 $50 \%$, 而不需要 的异构体只能丢弃或通过消旋化方法进行回收(Eq. 1). 动态动力学拆分能够克服上述不足 ${ }^{[26 ~ 28]}$, 其可以对仲 醇进行成酯拆分的同时, 实现对不需要的异构体进行原 位消旋，拆分与消旋的过程在同一条件下进行，该拆分 理论收率为 $100 \%$ (Scheme 1). 消旋化方法根据底物结 构的不同而有所差异, 主要有化学法 ${ }^{[29]}$ 和生物法两种 [30]. 动态动力学拆分在传统的化学拆分中, 已有大量案 例报道，如将其用于抗凝药氯吡格雷关键中间体 $(R)$-邻 氯苯甘氨酸的合成案例中 ${ }^{[31,32]}$.

上述介绍的动态动力学拆分, 不论是使用有机拆分 剂还是生物催化剂, 均是制备具有单一手性中心化合物 的方法，是否可以通过一步反应同时制备含两个或两个 
<smiles>[R]C(O)C(C)OC(C)=O</smiles><smiles>[R]C(O)C(C)OC(C)=O</smiles>

(S)-构型

图式 1 动态动力学拆分

Scheme 1 Dynamic kinetic resolution

以上手性中心的化合物? 在金属配体参与催化的动态 动力学拆分中, 已有大量相关案例报道 ${ }^{[33 \sim 35]}$. 而通过整 细胞或分离酶参与的动态动力学拆分案例也有少量报 道, 最早可追溯至 1976年, 研究者使用面包酵母还原氨 基酮类底物, 依底物结构不同, 整细胞表现出不同的立 体选择性. 随后研究者陆续报道, 通过使用含有羰基还 原酶的野生菌对类似反应进行研究, 对于部分底物羰基 还原酶显示出良好的立体选择性 ${ }^{[36]}$. 随后的二十年间, 类似的研究案例报道较少, 推测主要有三方面的原因: 一是该方法对酶催化的立体选择性的特性要求高, 符合 条件的酶难以直接从自然界获得; 二是受限于基因测序 与合成技术的发展滞后, 酶的篮选与分离表达研究工作 进展缓慢, 导致供研究的酶的类别较少; 三是有机合成 专家与生物催化专家缺乏有效的合作. 近十年来, 由于 DNA 测序与合成技术的突破与快速发展, 羰基还原酶 研究领域取得了长足的进步, 越来越多的野生型羰基还 原酶被鉴定、分离与表达 ${ }^{[37,38]}$; 同时，通过分子生物学、 计算机辅助的蛋白质定向进化技术对野生型酶进行改 造以适应工业化化学反应的要求已成为可能 ${ }^{[39 \sim 41]}$, 因 此将羰基还原酶与动态动力学拆分相结合展现出了潜 在的基础与应用前景.

\section{1 原理与研究案例}

\section{1 原理}

当羰基还原酶与动态动力学拆分结合, 我们便可经 一步还原反应制备含两个手性中心的醇类化合物，其原 理为: 通过羰基还原酶立体选择性地还原单一构型的潜 手性酮(如 $R$-构型), 同时另一构型的潜手性酮(如 $S$-构 型)通过羰基烯醇互变或其他条件, 实现 $\alpha$-位手性构型 的消旋, 还原与消旋在同一反应条件下进行, 实现高效
制备含两个手性中心仲醇的目的(Scheme 2). 此方法有 两个关键点：一是羰基还原酶对单一构型的潜手性酮的 立体选择性还原；二是消旋条件与还原条件的兼容性， 后面会加以详细论述.<smiles>[R]C(=O)C([R])[R]</smiles>

(S)-潜手性酮

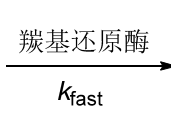

(1)

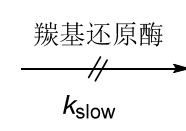<smiles>[R]C([R])C([R])O</smiles>

$(R, S)$-仲醇
图式 2 羰基还原酶参与的动态动力学拆分

Scheme 2 Dynamic kinetic resolution catalyzed by carbonyl reductases

本文重点对羰基还原酶参与的动态动力学拆分的 研究案例进行梳理，并结合实际工作，尝试对其研究方 法进行整理与分析, 为后续研究提供借鉴. 本综述主要 分为两部分：应用案例和研究方法.

\section{2 研究案例}

基于上述原理，从不同底物结构出发，应用该方法 可以制备结构多样的含有多手性中心的仲醇，包括烷烃 醇、卤代醇、硫醚醇、双醇和氨基醇(Scheme 3).<smiles>[R]C(=O)C([R])[R]</smiles>

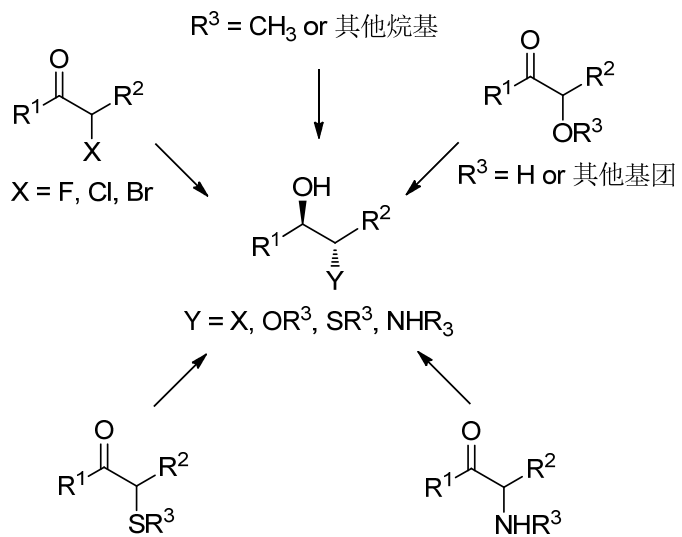

$\mathrm{R}^{3}=\mathrm{H}$ or 其他基团

$\mathrm{R}^{3}=\mathrm{H}$ or 其他基团

图式 3 不同酮类化合物的化学转化

Scheme 3 Transformation of different ketones

\subsection{1 制备烷烃醇}

当 $R^{1}$ 为非氢原子或基团, $R^{2}$ 为酯基、氧基、硝基或 其他吸电子基团, $\mathrm{R}^{3}$ 为烷烃基团时, 羰基还原酶催化还 
原可制备含两个手性中心碳的烷烃醇(Scheme 3). 羰基 还原酶动态不对称还原该类潜手性酮的研究案例较为 丰富.

2005 年 Smonou 及其团队 ${ }^{[42]}$ 在对底物酮酯与双酮等 底物进行立体选择性还原时, 篮选了 20 种分离的羰基 还原酶，当底物结构为 $\alpha$-甲基乙酰乙酸酯时, KRED-102 表现出显著的立体选择性, ee $>99 \%, d e>99 \%$ 且转化率 为 $100 \%$. 当 $\alpha$ 位的基团变化时, 不同的羰基还原酶表现 出不同的立体选择性(Scheme 4).

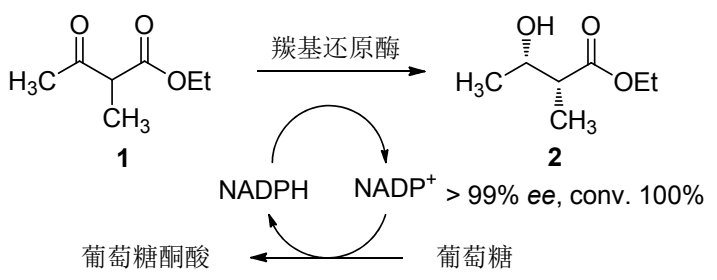

图式 4 化合物 $\mathbf{1}$ 的还原

Scheme4 Reduction of compound 1

金属配体参与的不对称催化合成中, 对双酮进行区 域与立体选择性还原是具有挑战性的研究领域. 2009 年 Lüdeke 等 ${ }^{[43]}$ 尝试开展对双酮进行羰基还原酶参与的动 态动力学拆分的应用研究, 期望实现区域与立体选择性 地制备含两手性中心碳的仲醇. 研究者通过篮选商业化 和自主构建的羰基还原酶, 对底物进行还原, 结果发现 不同的酶对相同的底物可以实现定向合成四个异构体 中的三个, 实现了双酮的区域选择性还原(Scheme 5).

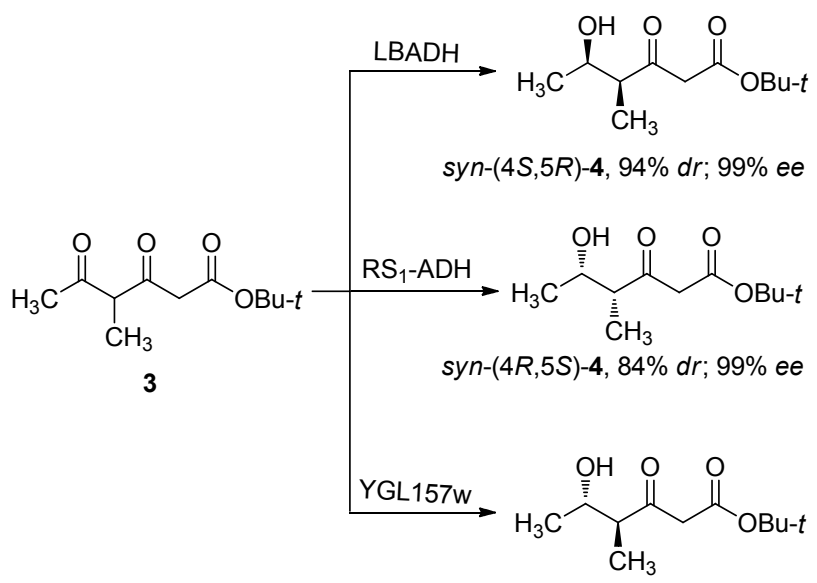

anti-(4S,5S)-4, $86 \%$ dr; $90 \%$ ee

图式 5 化合物 $\mathbf{3}$ 的立体还原

Scheme 5 Stereoselective reduction of compound $\mathbf{3}$

2005 年 Yasohara 等 ${ }^{[44]}$ 研究者在专利中报道了碳青 霉烯类抗生素通用中间体 4-AA 的化学-酶法路线. 4-AA 化学结构中含三个手性碳, 其中两个是通过动态还原动 力学拆分的方法实现的. 研究者经篮选发现短乳酸杆菌
(Lactobacillus brevis)醇脱氢酶显示高的立体选择性, ee $>99 \%, d e=92 \%$. 随后 2007 年, Codexis 公司报道对短 乳酸杆菌(Lactobacillus brevis)醇脱氢酶进行蛋白质改 造, 以提高酶的催化效率、底物浓度、溶剂、 $\mathrm{pH}$ 与温度 耐受性为多目标, 经多轮进化, 最终篎选到满足工业化 要求的多个序列酶. 在最优实施例中使用 KRED10酶还 原时，反应底物浓度高达 $300 \mathrm{~g} / \mathrm{L}$, 反应转化率 $>96 \%$, 异丙醇浓度达 $50 \%$, 生物转化一步还原形成两个手性中 心, $e e>99 \%, d e>99 \%{ }^{[45]}$. 酶法的突破为实现该路线的 产业化开发提供了良好的基础(Scheme 6).
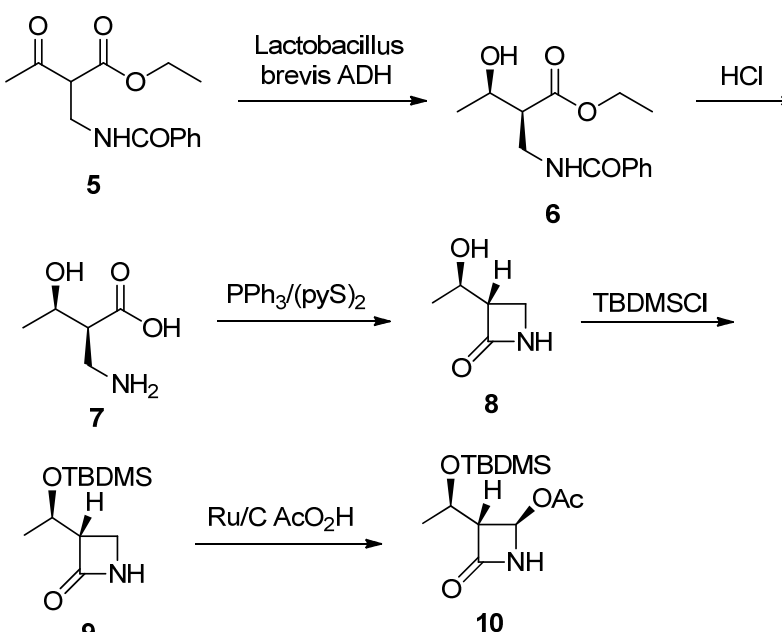

10

图式 6 4-AA 的合成路线

Scheme 6 Route of 4-AA

2013 年 Gotor 及其团队 ${ }^{[46]}$ 通过使用还原酶 ADH-A, 经动态还原动力学拆分制得手性醇, 后经氰基水解, 关 环成酯制备苯并内酯环，其中还原反应具有显著的立体 选择性, $e e>99 \%, d r>99 \%$. 该研究对羰基还原酶参与 的动态动力学拆分技术提供了较完整的研究思路, 研究 人员初始时发现，该还原反应仅表现为动力学拆分的特 点，即收率 $<50 \%$ ，随后研究者调整思路，尝试对不需 要异构体潜手性酮进行消旋化研究，由于底物结构中羰 基 $\alpha-\mathrm{H}$ 表现一定酸性, 经笁选发现向反应体系中加入 $1 \% \sim 3 \%$ 三乙胺, $\mathrm{pH}$ 约为 8 时，可实现其消旋，最后将消 旋化与还原在同一反应体系下进行，实现该步的动态动 力学拆分(Scheme 7).

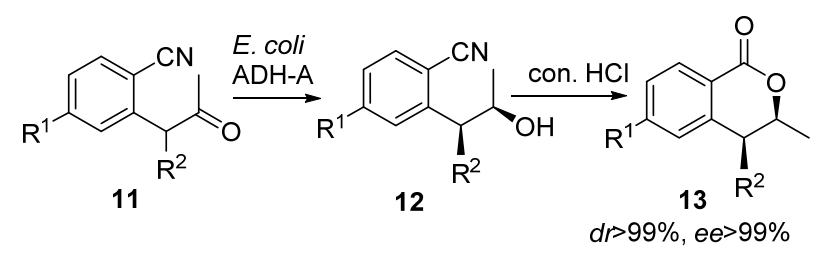

图式 7 化合物 $\mathbf{1 3}$ 的合成

Scheme 7 Synthesis of compound $\mathbf{1 3}$ 
同样地, 2016 年由 Merck 公司、Codexis 公司与上 海合全公司组成的研究团队, 为大量供应在研药物 MK-8666 三期临床实验的使用, 成功开发了一条羰基 还原酶参与的动态动力学拆分路线. 该路线主要集中对 中间体 19 进行还原研究, 研究者希望通过动态动力学 拆分制备两个手性碳的仲醇 trans-20 (Schemes 8、9). 底 物 19 仅具有一个酮羰基, 其 $\alpha-\mathrm{H}$ 的酸性预测值为 $\mathrm{p} K_{\mathrm{a}} 18.5$, 需要在较强的碱性条件才能实现消旋, 所以要 求酶对碱性条件的耐受性更强. 研究者对金属配体与羰 基还原酶进行了篮选, 金属配体催化条件下主要获得顺 式产物，而不同的酶可制备不同构型的顺或反式产物. 初篮中发现 KRED-208 表现一定选择性 trans-20/cis$20 \mathbf{a}=7 / 3$, 后经六轮蛋白质定向进化, 获得具有显著立 体选择性与高催化活性的羰基还原 KRED264, 转化的 底物浓度提至 $50 \mathrm{~g} / \mathrm{L}$ 、立体选择性提高至 $e e>99 \%$ 、 $d r>30: 1$, 反应在 $\mathrm{pH}=9,50{ }^{\circ} \mathrm{C}$ 条件下便可实现底物 的消旋 ${ }^{[47]}$.

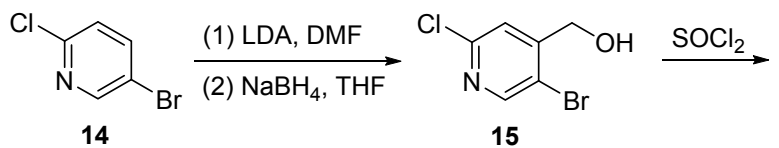

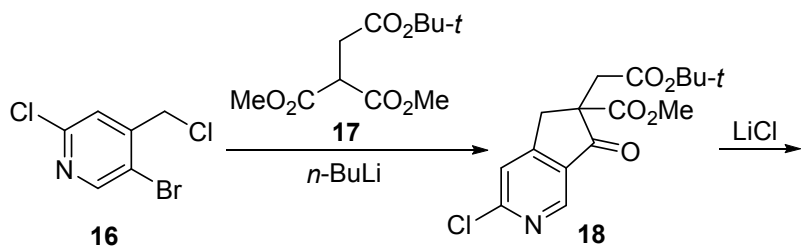<smiles>CCCCOC(=O)CC1Cc2cc(Cl)ncc2C1=O</smiles>

图式 8 trans-20 的合成路线

Scheme 8 Synthetic route of trans-20<smiles>CCCCCC(=O)OCC1Cc2cc(Cl)ncc2C1=O</smiles>

19<smiles>CCCOC(=O)C[C@H]1Cc2cc(Cl)ncc2C1O</smiles>

trans-20c

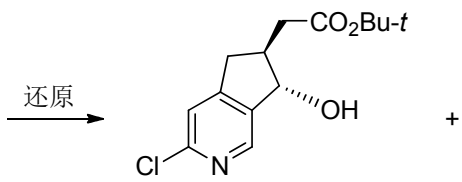

trans-20

$>30: 1 d r ;>99 \%$ ee

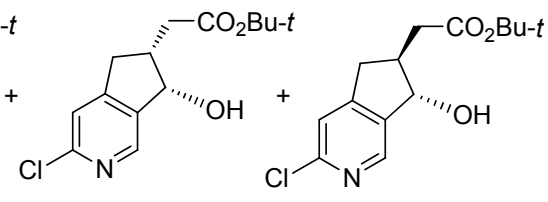

cis-20b

图式 9 化合物 19 的生物催化还原

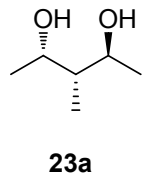

$88 \%$ yield $>99 \%$ ee $>99 \%$ de
Dimitris 团队 ${ }^{[48]}$ 报道了羰基还原酶对双酮羰基化合 物进行区域与立体选择性还原制备含 3 个手性中心碳的 双醇的方法. 第一阶段篮选到 KRED-102 酶经动态动力 学拆分, 区域选择性地还原一个酮羰基, 先制备两个手 性中心的羟酩，第二阶段䇥选到 KRED-101 与 KREDA1B 酶对第二个羰基进行还原形成第三个手性中心, 进 而制备双醇(Scheme 10). 研究者发现第一阶段的反应 转化完全且无副反应发生，所以将两个阶段在同一反应 缓冲液内进行，实现一锅法制备含三手性中心的双醇， 该法充分表现出酶催化的绿色与高效. 最后研究者对反 应的底物谱进行扩展，该法表现一定的通用性。

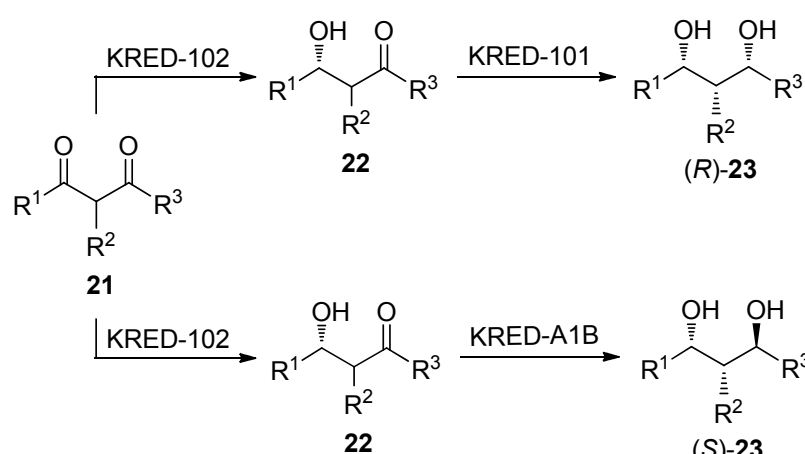

$(S)-23$
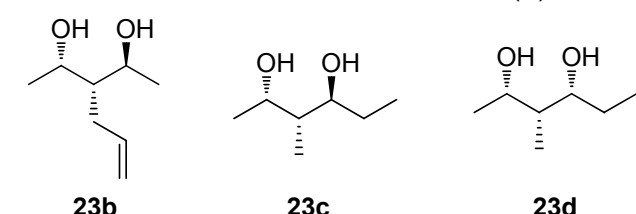

23d

$85 \%$ yield $>99 \%$ ee $96 \%$ de

图式 10 两次立体还原化合物 21

Scheme 10 Double reductions of compound 21

上述案例主要围绕脂肪和芳香酮酯的还原, 对于环 状的酮酯进行动态还原动力学拆分同样有相关的案例 报道. Danchet 等 ${ }^{[99]}$ 开发了整细胞催化还原 7、8 元环酮 酯的动态动力学拆分方法. 作者筛选了 11 种野生菌, 结 果表明对于 $7 、 8$ 元环表现出不同的立体选择性. 克勒克 酵母菌(Kloekera magna) 对于 7 元环表现显著的立体选 择性, $e e=94 \%, d e=100 \%$ (Eq. 2); 然而对于 8 元环，却 难以催化反应的进行. 令人惊喜的是球孢白偪菌 (Beauveria bassiana)与灰蓝毛霉菌(Mucorgriseocyanus) 可以高立体选择性的催化底物(Eq. 3).

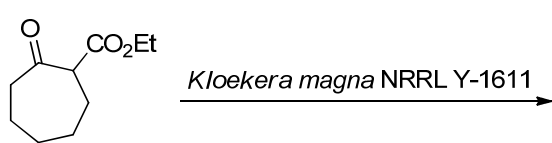

24

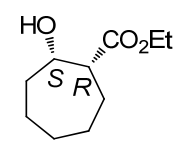

25

ee $94 \%$, de $100 \%$

Scheme 9 Reduction of compound 19 by biocatalysis 


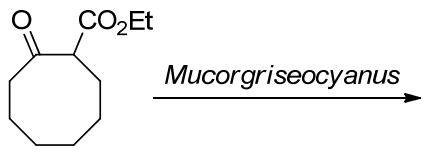

26

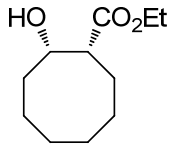

27
Merck 公司研发人员 ${ }^{[50]}$ 报道了 $\alpha, \beta$-不饱和酮酯的还 原方法, 包括金属配体催化氢化与羰基还原酶催化. 对 底物 28 进行动态动力学拆分时, 金属配体催化氢化可 以高对映选择性地制备 29, 但缺乏非对映选择性. 随后 Kosjek 团队使用嶡基还原酶进行还原, 发现部分酶表现 出良好的对映选择性 $e e>99 \%$, 具有一定的非对映选择 性 $d e=60 \%$. 接着研究人员改变底物结构, 比较不同酶 催化的非对映选择性, 发现将乙酯改为甲酷即能将非对 映选择性 $d e$ 值由 $60 \%$ 提高至 $95 \%$ (Scheme 11). 该案例 有两点启示: 一是可以通过对 $\alpha, \beta$-不饱和酮酯的动态不 对称还原制备含两个手性中心碳的烯丙醇; 二是酶与底 物之间立体选择性关系既可以通过对酶的改造来实现， 也可以通过对底物结构的变化来影响.

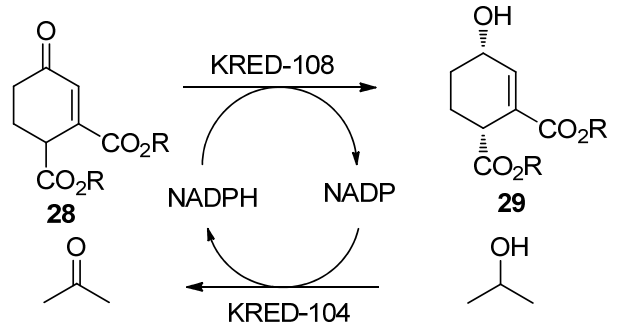

图式 11 化合物 $\mathbf{2 8}$ 的还原

Scheme 11 Reduction of compound 28

\subsection{2 制备 $\alpha$-卤代醇}

当 $R^{1}$ 为非氢原子或基团, $R^{2}$ 为酯基、氰基、硝基或 其他吸电子基, $\mathrm{R}^{3}$ 为卤素时, 羰基还原酶可还原底物制 备含两个手性中心碳的卤代仲醇(Scheme 3). Musa 等 ${ }^{[51]}$ 的研究工作说明可以通过理性设计与定点突变技术实 现对酶的改造, 进一步扩大酶的底物谱与提高催化活 性. 作者对来自于嗜热厌氧乙醇杆菌 (Thermoanaerobacter ethanolicus ADH, TeSADH)进行蛋白质改造, 改 变其催化口袋大小, 改变其催化的立体选择性, 笁选获 得突变株 $\mathrm{W} 110 \mathrm{~A}$, 实现了对 $\alpha$-氯代酮衍生物不对称的 动态还原, 获得 $e e=99 \%, d e=84 \%$ (Eq. 4).<smiles>O=C(Cl)C(Cl)Cc1ccc(Cl)cc1</smiles>

30<smiles>C[C@@H](O)[C@@H](Cl)Cc1ccc(Cl)cc1</smiles>

31
$83 \%$ yield, $84 \%$ de; $99 \%$ ee 线. 首先对起始物料 4 进行氯代制得氯代酮酯化合物

33, 然后篎选了分离的羰基还原酶，其中 YDL124W 酶 可高立体选择性地制得 34, 收率 $91 \%, e e>98 \%, d e>$ $98 \%$. 由于底物与产物对酶活性有明显的抑制作用，底 物浓度仅能达到约 $1.3 \mathrm{~g} / \mathrm{L}$. 另外文献报道反应在 $\mathrm{pH} 5.6$ 条件下进行，说明底物易于消旋. 然后化合物 34 经环氧 化, 苯异氧酸酯关环、水解制得侧链 37 (Scheme 12). 该 路线设计简洁, 原子经济性高. 由于被还原底物的分子 结构易于消旋，如果尝试更多篮选工作或对现有酶进行 改造, 有望克服现有路线中底物浓度较低的不足.

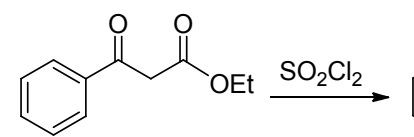

32<smiles>CCOC(=O)[C@H](Cl)[C@H](O)c1ccccc1</smiles>

34<smiles>CCOC(=O)C(Cl)C(=O)c1ccccc1</smiles>

33

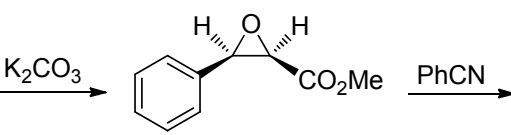

35

yield: $91 \%$, ee $>98 \%$, de $>98 \%$<smiles>CC(=O)C1OC(c2ccccc2)=N[C@H]1c1ccccc1</smiles>

36<smiles>CCOC(=O)[C@@H](O)[C@H](N)c1ccccc1</smiles>

37
图式 12 化合物 37 的合成路线

Scheme 12 Route of synthesizing compound 37

2011 年 Marocco 等 ${ }^{[53]}$ 报道了一条磷霉素的化学-酶 法路线，该路线关键步骤为应用羰基还原酶经动态动力 学拆分制备化合物 syn-(1R,2S)-39 (Scheme 13). 研究人 员筛选了 20 种羰基还原酶, 其中三种酶 YBR149W、 YHR104W、YDR368W 表现出高的立体选择性, ee> $99 \%, d e>90 \%$, 转化率 $>98 \%$, 底物浓度约 $1 \mathrm{~g} / \mathrm{L}$. 反应 条件 $\mathrm{pH} 7.0$ 与温度 $28{ }^{\circ} \mathrm{C}$, 表明化合物 $\mathbf{3 8}$ 易于消旋. 使 用 YDR368W 制备 syn-(1R,2S)-39 产物后，再经两步反 应制备磷霉素. 较现有传统拆分工艺，该路线简洁，有 一定应用开发前景(Scheme 14).

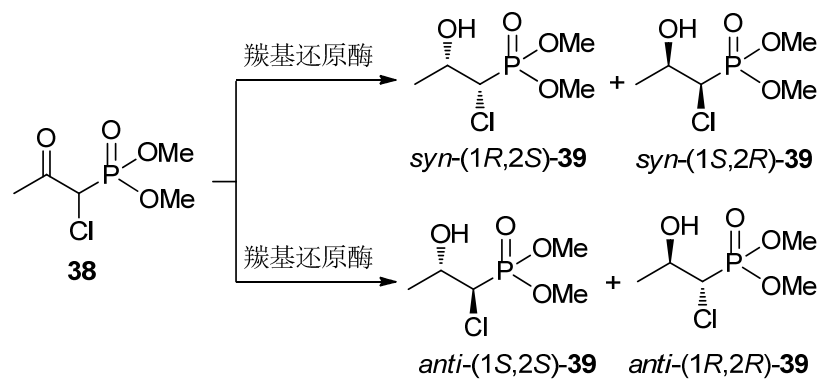

图式 13 化合物 $\mathbf{3 8}$ 的生物还原

Scheme 13 Bioreduction of compound 38 
表 4 筛选结果

Table 4 Results of screening

\begin{tabular}{cccc}
\hline No. & 催化剂 & syn-(1R,2S)-39 & anti-(1S,2S)-39 \\
\hline 1 & YOL151w & ND & $64 \% d e$ \\
2 & YGL157w & $12 \% d e$ & ND \\
3 & YGL039w & ND & $56 \% d e$ \\
4 & YNL331c & $79 \% d e$ & ND \\
5 & YOR120w & $77 \% d e$ & ND \\
6 & YBR149w & $95 \% d e$ & ND \\
7 & YHR104w & ND & $92 \% d e$ \\
8 & YDR368w & $95 \% d e$ & ND \\
9 & 面包酵母 & $18 \% d e$ & ND \\
\hline
\end{tabular}

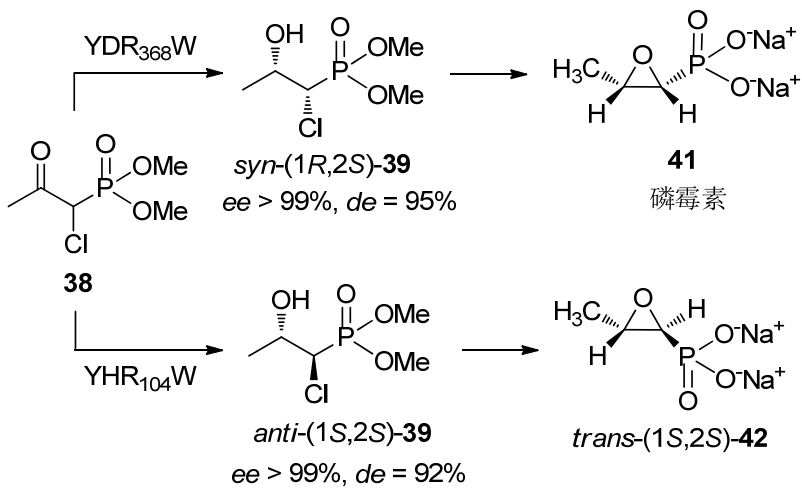

图式 14 磷需素的合成路线

Scheme 14 Route of fosfomycin

\subsection{3 制备双醇及其衍生物}

当 $R^{1}$ 为非氢原子或基团, $R^{2}$ 为酯基、氰基、硝基或 其他吸电子基, $\mathrm{R}^{3}$ 为羟基、烷氧基或烷颈基时, 羰基还 原酶可以通过立体选择性的还原该类潜手性酮制备含 两个手性中心碳的双醇或醇醚衍生物(Scheme 3). 由于 着基酮底物较难制备, 因此现有文献报道案例较少.

Perrone 等 ${ }^{[54]}$ 研究者对底物芳醚酮酯类化合物 43 进 行动态动力学拆分研究. 研究人员篮选了 5 种野生菌, 进行整细胞催化, 发现静息细胞比生长细胞的催化效率 高. 作者幸运的篮选到野生菌马克思克鲁维酵母 (Kluyveromyces marxianus $)$ 可以高立体选择性 $(e e=97 \%, d e>$ 99\%)制备 syn-(2R,3S)-44 (Eq. 5).
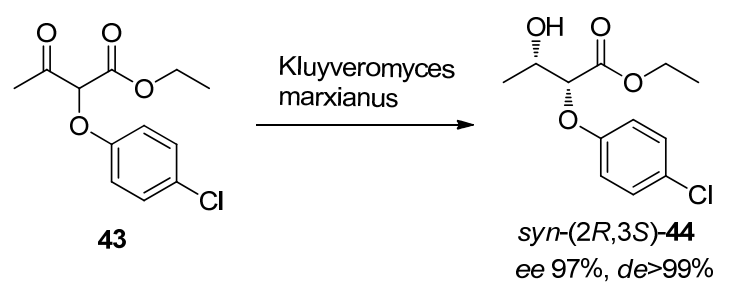

\subsection{4 制备氨基醇}

当 $R^{1}$ 为非氢原子或基团, $R^{2}$ 为酯基、氰基、硝基或 其他吸电子基, $\mathrm{R}^{3}$ 为氨基或其衍生基团时, 羰基还原酶
可以通过立体选择性的还原该类潜手性酮制备含两个 手性中心碳的氨基醇及其衍生物(Scheme 3).

1993 年 Ramesh 才队 ${ }^{[55]}$ 在合成紫杉醇侧链时, 通过 对含有羰基还原酶的微生物进行大量篮选，最终获得一 株具有良好立体选择性的多型汉逊酵母(Hansenula polymorpha) SC13865, ee $>99 \%$. 然而对于顺反立体异 构的区域选择性 $(d e>50 \%)$ 尚不理想(Eq. 6). 分析原因 可能是微生物体内存在多种羰基还原酶，干扰了彼此的 立体选择性，如果进行单酶的离体表达，还原效果可能 会有改观.

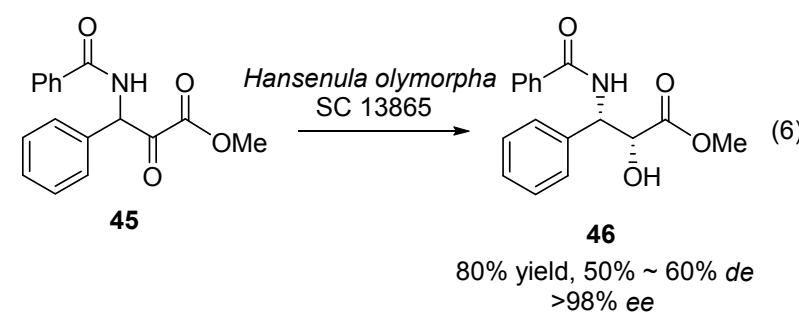

Kataoka 等 ${ }^{[56,57]}$ 研究人员设计了一条伪麻黄碱的生 物制备方法，即以氨基酮盐酸盐为底物，广泛笁选羰基 还原酶, 经动态动力学拆分高立体选择性的制备伪麻黄 碱. 该团队 2006 年从圆平球菌(Rhodococcus erythropolis) MAK154 篎选到氨基丙醇脱氢酶，该羰基还原酶表 现良好的立体选择性, ee $>99 \%, d e>99 \%$ (Scheme 15). 然而该酶表现明显的底物与产物抑制现象，底物浓度仅 为 $40 \mathrm{~g} / \mathrm{L}$. 随后 2011 年, Kataoka 等 ${ }^{[58]}$ 针对野生酶存在的 缺陷，对其开展了蛋白质改造工作，最终笁选到催化活 性更优的突变体：对底物与产物的耐受性显著提高; 底 物浓度可提高至 $100 \mathrm{~g} / \mathrm{L}$. 该制备方法表现出潜在的工 业化应用前景.

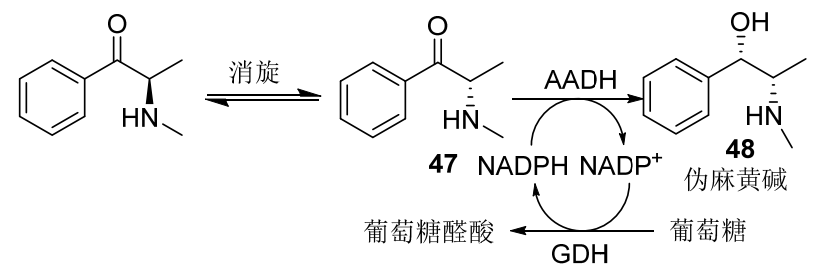

图式 15 化合物 $\mathbf{4 7}$ 的生物还原

Scheme 15 Bioreduction of compound 47

2013 年为临床提供候选药物 49 时, Merck 公司研发 人员 ${ }^{[59]}$ 成功开发一条对关键底物氨基酮酯进行动态动 力学还原的合成路线. 研究人员通过对自有酶库进行篮 选获得具有潜在开发前景的羰基还原酶 CDX-901. 在 放大过程中由于底物溶解性低的原因，导致反应转化率 降低，随后研究者对该酶进行改造，提高其对有机溶剂 的耐受性. 经过笁选获得突变体 CDX-108, 转化反应底 物浓度提高至 $100 \mathrm{~g} / \mathrm{L}$, 转化率 $>95 \%$, 产物 $e e>99 \%$, 
de $>99 \%$ (Eq. 7).<smiles>Nc1ccc(CC2CCCC2C(O)c2ccccc2)cc1</smiles>

49<smiles>COC(=O)C(Nc1ccccc1)C(=O)O</smiles>

50

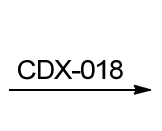<smiles>COC(=O)C(O)C(N)c1ccccc1</smiles>

51

$92 \%$ yield $99 \%$ de; $99 \%$ ee

\section{2 .5 其他}

羰基还原酶能够还原醛基为羟基的研究案例较少. 因为该方法不涉及手性中心的变化, 而现有的化学还原 方法选择较多, 如硼系列的还原体系与氢气还原体系. Friest 等 ${ }^{[60]}$ 报道了可以高立体选择性的还原醛基的醇脱 氢酶 SsADH10, 该酶最大耐受温度高达 $80{ }^{\circ} \mathrm{C}$. 将该脱 氢酶与动态动力学拆分结合, 为制备非甾体抗炎药 $(S)$ 布洛芬提供了新颖的研究思路(Scheme 16).

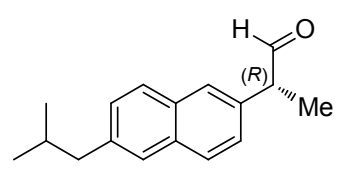

(R)-52

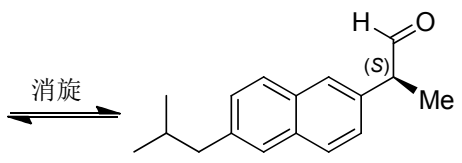

(S)-52
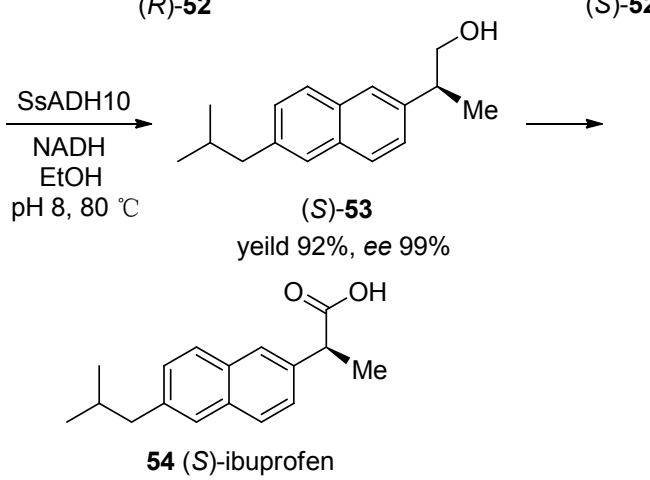

图式 16 布洛芬的合成

Scheme 16 Synthesis of (S)-Ibuprofen

\section{2 研究方法}

由前述案例分析知, 羰基还原酶参与的动态动力学 拆分包括三部分: 一是羰基还原酶对需要的单一手性异 构体潜手性酮的立体选择性还原; 二是对不需要的单一 手性异构体潜手性酮的消旋化; 三是还原与消旋两部分 的兼容与平衡.

首先, 在确定并完成底物的合成后, 需要去寻找满 足研究要求的羰基还原酶. 研发早期主要有三条途径: 一是阅读分析文献, 根据底物相似性, 通过克隆表达,
自制羰基还原酶，供筛选使用; 二是篮选已建立的羰基 还原酶库, 该库可以为内部积累亦可为外部资源, 包括 拥有商业化酶库的企业或高校团队，三是从自然界中以 目标底物为模板, 建立篮选模型, 篮选含羰基还原酶的 野生菌. 羰基还原酶已有大量的工业化案例报道, 企业 酶库中多为已实现产业化品种的酶, 工业化前景较好, 可优先尝试. 若将羰基还原酶应用于动态动力学拆分, 须对酶的特性有两点要求: 一是高的对映选择性( $e e>$ 99\%); 二是高的非对映选择性 ( $d e>95 \%)$. 对映选择性 易于实现, 非对映选择性要求酶在还原羰基的同时对潜 手性酮 $\alpha$-位的手性异构体 $R$ 或 $S$ 构型具有相对映的立体 选择性. 因此, 在前三条途径无法篮选到同时满足上述 两种立体选择性的情况下, 研究者可以优先选择具有高 对映选择性( $e e>99 \%$ ), 但具有部分非对映选择性(如 $d e$ $>80 \%$ ) 的羰基还原酶作为初始模板, 通过蛋白质改造 的手段, 对酶进行再设计, 建立突变体库, 进行有效篮 选, 以期获得满足立体选择性要求的酶. 目前, 该类技 术已经有大量的研究案例报道, 如上述案例卤代醇 ${ }^{[51]}$ 和氨基醇 ${ }^{[59}$ 均涉及到对酶进行改造.

其次, 对不需要异构体潜手性酮的消旋化研究. 2013 年 Gotor 团队 $^{[46]}$ 的研究思路就涉及该部分内容 (Scheme 7). 研究者通过筛选不同碱性条件对不需要的 潜手性酮进行消旋, 发现在 $1 \%$ ～3\%三乙胺条件下，可 将底物进行消旋. 上述案例消旋化的原理均在碱性条件 下, 通过宸基烯醇互变对 $\alpha$-位手性中心进行消旋化, 所 以 $\alpha-\mathrm{H}$ 的酸性强弱就直接决定了消旋化的难易. Applegate 等 ${ }^{[62]}$ 总结动态还原动力学拆分案例, 发现通常 $\alpha-\mathrm{H}$ 的 $\mathrm{p} K_{\mathrm{a}}$ 值在 7.5 12.5 $\left(\mathrm{H}_{2} \mathrm{O}\right.$ 或 DMSO) 易于消旋化. 但是, 这并非教条, 2016 年 Merck 团队 ${ }^{[47]}$ 开发的路线中, 底物 预测的 $\mathrm{p} K_{\mathrm{a}}$ 值为 18 , 仍可在 $\mathrm{pH} 9$ 的条件下实现动态动 力学拆分 (Scheme 9). 碱性消旋在前述案例中占绝大多 数. 但其他条件如酸性条件、加热、金属离子催化或通 过氨基与酫形成席夫碱进行消旋，上述各法均值得探 究.

最后将羰基还原酶催化条件与消旋化条件相结合, 实现两者反应速率的平衡. 两者结合过程中, 可能会出 现一种不利情况: 即消旋速度显著慢于还原的速度, 此 时容易导致羰基还原酶识别不需要的潜手性酮进行还 原, 从而降低非对映选择性. 因此当非对映选择性在多 批次反应中存在差异时, 可以通过调整消旋的速度, 以 与酶还原速率相匹配. 这样便可在同一反应容器内进 行，同时高效构建两个手性中心碳.

\section{3 总结与展望}

本文主要对羰基还原酶参与的动态动力学拆分的 
应用案例进行了梳理与分析, 介绍该技术的应用进展, 并尝试提供一种可行的研究思路: 首先是酶的笁选与改 造; 其次是消旋化研究; 最后实现两者反应速率的平衡. 将该方法应用于不同的底物可制备不同类型的手性化 合物，如具有多手性中心的氨基醇、双醇、三醇、手性 烷烃醇及羟基氨基酸等(Scheme 17), 该法在有机合成 官能团转化中表现良好的应用前景.

羰基还原酶催化的动态动力学拆分与金属配体催 化的动态动力学拆分形成优势互补, 但具有自身四个特 点: 羰基还原酶具有一定成本优势; 转化反应在水中进 行; 反应条件温和; 催化剂的选择范围广, 商业化的羰 基还原酶种类丰富以及酶的改造手段逐渐成熟. 一经篎 选到合适的羰基还原酶, 便可快速地实现酶转化工艺的 产业化. 将生物催化与传统有机化学反应相结合, 可以 更丰富有机反应的类型, 并产生更为巧妙的化学结果. 如对于本文动态动力学拆分方法, 若将酶的范围由羰基 还原酶扩展至转氨酶, 并与动态动力学拆分结合, 便可
经一步转氨反应，制备含两个手性中心碳的仲胺，已有 相关案例报道 ${ }^{[63]}$, 该法是传统金属配体催化难以实现 的，同理我们可以依据不同化学反应特点，与不同种类 的生物催化剂进行有效结合, 因此有机合成专家可以更 加广泛的视角去设计适宜工业化生产的合成路线. 要实 现化学合成与酶催化的有效结合, 首先需要有机合成专 家建立基于生物催化基元反应的逆合成分析意识，其次 需要跨学科合作，包括生物催化、有机合成以及蛋白改 造等领域. 随着新酶数量与种类的不断增长, 酶基因挖 掘技术的进步与蛋白改造技术的逐渐成熟, 生物催化剂 将会与金属配体催化剂互补协作在手性药物的制备中 展现更为广泛的基础与应用价值.

致谢 中国科学院上海生命科院研究院湖州工业生物 技术中心陶荣盛副主任，中国科学院天津工业生物技术 研究所祝俊研究员提供的宝贵意见.

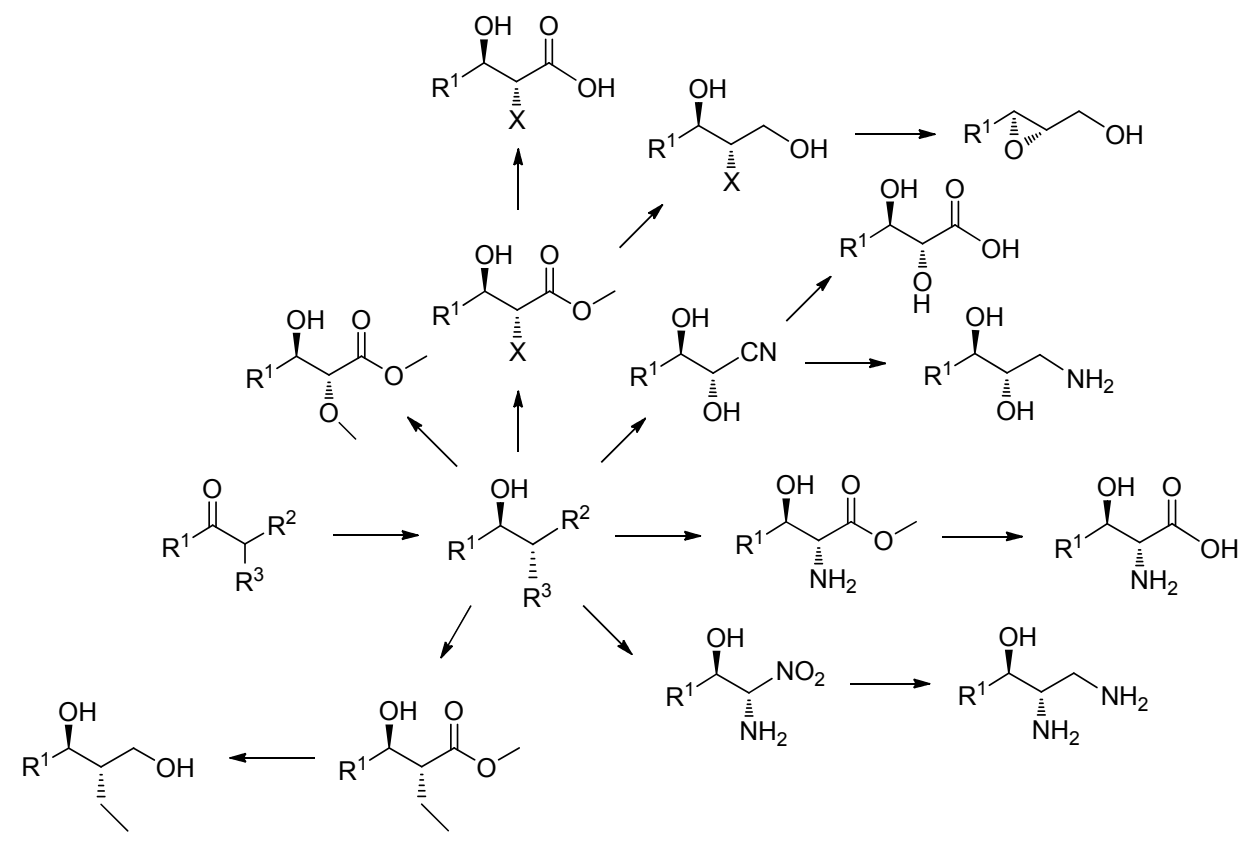

图式 17 官能团的转化

Scheme 17 Transformation of functional groups

\section{References}

[1] Lin, G.-Q.; Li, M.-Y.; Chen, Y.-Q.; Sun, X.-W.; Chen, X.-Z. Chiral Synthesis-Asymmetric Reactions and Its Application, Science Press, Beijing, 2010, p. 584 (in Chinese).

(林国强, 李明月, 陈耀全, 孙兴文, 陈兴滋, 手性合成一不对称 反应及其应用(第四版), 科学出版社, 北京, 2010, p. 584.)

[2] Straathof, A. J.; Panke, S.; Schmid, A. Curr. Opin. Biotechnol. 2002, 13, 548 .

[3] Tao, J.-H.; Lin, G.-Q.; Liese, A. Biocatalysis for the Pharmaceutical Industry Discovery, Development and Manufaturing, Chemical Industry Press, Beijing, 2010, p. 3 (in Chinses).

(陶军华, 林国强, 安德烈亚斯・李斯, 生物催化在制药工业的应 用一一发现、开发与生产, 化学工业出版社, 北京, 2010, p. 3.)
[4] Truppo, M. D.; Pollard, D.; Devine, P. Org. Lett. 2007, 9, 335

[5] Kavanagh, K. L.; Klimacek, M.; Nidetzky, B.; Wilson, D. K. Biochemistry 2002, 41, 8785.

[6] Xu, Z.; Jing, K.; Liu, Y.; Cen, P. J. Ind. Microbiol. Biotechnol. 2007, 1, 83.

[7] Inoue, K.; Makino, Y.; Itoh, N. Appl. Environ. Microb. 2005, 7, 3633.

[8] Ma, S. J.; Gruber, J.; Davis, C.; Newman, L.; Gray, D.; Wang, A.; Grate, J.; Huisman, G. W.; Sheldon, R. A. Green Chem. 2010, 12, 81.

[9] Liang, J.; Lalonde, J.; Borup, B.; Mitchell, V.; Mundorff, E.; Trinh, N.; Kochrekar, D. A.; Cherat, R. N.; Pai, G. G. Org. Process Res. Dev. 2010, 193.

[10] Nurit, P.; Ayelet, M. WO 2008151324. 2008. 
[11] Bong, Y. K.; Vogel, M.; Collier, S. J.; Mitchell, V.; Mavinahalli, J. WO 2011005527, 2010.

[12] Li, H. G.; Moncecchi, J.; Truppo, M. D. Org. Pro. Res. Dev. 2015, 19, 695 .

[13] Savile, C.; Gruber, J. M.; Mundorff, E.; Huisman, G. W.; Collier, S. J. WO 20100252, 2009.

[14] Isotani, K.; Kurokawa, J. J.; Itoh, N. Int. J. Mol. Sci. 2012, 13, 13542.

[15] Zheng, W.-X.; Xu, G.-C.; Huang, L.; Pan, J.; Yu, H.-L.; Xu, J.-H. Org. Lett. 2013, 19, 4917

[16] Zhang, F.-L.; Ni, G.-W. Chen, S.-X.; Ju, D.-W.; Tang, J.-W.; Tan, Z.-M.; Zou, J.; Guo, X.; Wang, Z.-W. CN 201611008108, 2016.

[17] Zhang, F.-J.; Liu, Y.; Peng, X.-Q.; Guo, C.; Wu, Z.-L. Appl. Microbiol. Biotechnol. 2016, 5,1 .

[18] Liang, J.; Jenne, S. J.; Mundorff, E.; Ching, C.; Gruber, J. M.; Krebber, A.; Huisman, G. W. WO 2009036404, 2008.

[19] Xu, G.-P.; Wang, H.-B.; Wu, Z.-L. Process Biochem. 2016, 51, 881.

[20] Tao, J.-H.; Li, G.-Q.; Liese, A. Biocatalysis for the Pharmaceutical Industry-Discovery, Development and Manufaturing, Chemical Industry Press, Beijing, 2010, p. 121 (in Chinese).

(陶军华, 林国强, 安德烈亚斯・李斯, 生物催化在制药工业的 应用——发现、开发与生产, 化学工业出版社, 北京, 2010, p. 121.)

[21] Luetz, S.; Giver, L.; Lalonde, J. Engineered Enzymes for Chemical Production. Biotechnology and Bioengineering, 2008, 4, 647.

[22] Tufvesson, P.; Lima-Ramos, J.; Nordblad, M.; Woodley, J. M. Org. Process. Res. Dev. 2011, 15, 266.

[23] Xin, L. H.; Nicholas, W.; Hans, I.; Vera, J.; Zhang, H. M.; Koenig, S. G.; Gossselin, Francis. Org. Process Res. Dev. 2017, 3, 387.

[24] Hou, X. P.; Zhang, H. P.; Chen, B. C. Guo, Z. W.; Singh, A.; Goswami, A.; Gilmore, J. L.; Sheppeck, J. E.; Dyckman, A. J. Carter, P. H.; Mathur, A. Org. Process Res. Dev. 2017, 2, 200.

[25] Ginotra, S. K. J.; Friest, A.; Berkowitz, D. B. Org. Lett. 2012, 14 , 968

[26] Pellissier, H. Adv. Synth. Catal. 2011, 353, 659

[27] Cheng, Y.-M.; Xu, G.; Wu, J.-P.; Yang, L.-R. Chin. J. Org. Chem. 2010, 31, 1695 (in Chinese). (程咏梅, 徐刚, 吴坚平, 杨丽蓉, 有机化学, 2010, 31, 1695.)

[28] Zhang, Z.-H.; Liu, Q.-B. Chin. J. Org. Chem. 2005, 25, 780 (in Chinese). (张占辉, 刘庆涁, 有机化学, 2005, 25, 780.)

[29] Larsson A. L. E.; Persson, B. A.; Bäckvall, J.-E. Angew. Chem., Int. Ed. 1997, 36, 1211.

[30] Sakulsombat, M.; Vongvilai, P.; Ramström, O. Chem.-Eur. J. 2014, 20, 11322 .

[31] Lhum, M. D.; Toulouse, J. R. US 5204469, 1991.

[32] Reddy, B. S. WO 2006003671, 2004.

[33] Wang, X.-L.; Xu, L.-J.; Yan, L.-J.; Wang, H.-F.; Han, S.; Wu, Y.; Chen, F. Tetrahedron 2016, 72, 1787.

[34] Sayo, N.; Satio, T.; Okeda, Y.; Nagashima, H.; Kumobayashi, H. US 4981992, 1991.

[35] Li, X.-M.; Tao, X.-M.; Ma, X.; Li, W.-F.; Zhao, M.-M.; Xie, X.-M.; Ayad, T.; Ratovelomanana-Vidal, V.; Zhang, Z.-G. Tetrahedron 2013, 34, 7152 .

[36] Deol, B. S.; Ridley, D.; Simpson, G. Aust. J. Chem. 1976, 29, 2459.

[37] Gamenara, D.; Sevane, G. A.; Mendez, P. S.; Maria, P. D. Redox Biocatalysis, Wiley, New Jewery 2013, Chapter 3.

[38] Faber, K.; Fessner, W. D.; Turner, N. J. Biocatalysis in Organic Synthesis 2, Georg Thieme Verlag KG, New York, 2015, pp. 421 458.
[39] Bornscheuer, U. T.; Huisman, G. W.; Kazlauskas, R. J.; Lutz, S.; Moore, J. C.; Robins, K. Nature 2012, 485, 185

[40] Savile, C. K.; Janey, J. M.; Mundorff, E. C.; Moore, J. C.; Sarena, T.; Jarvis, W. R.; Colbeck, J. C.; Krebber, A.; Fleitz, F. J.; Brands, J.; Devine, P. N.; Huisman, G. W.; Hughes, G. J. Science 2010, 329, 305 .

[41] Zheng, M.-M.; Chen, K.-C.; Wang, R.-F.; Li, H.; Li, C.-X.; Xu, J.-H. J. Agric. Food Chem. 2017, 6, 1178 .

[42] Kalaitzakis, D.; Rozzell, J. D.; Kambourakis, S.; Smonou, L. Org. Lett. 2005, 7, 4799

[43] Lüdeke, S.; Richter, M.; Müller, M. Adv. Synth. Catal. 2009, 351, 253.

[44] Yasohara, Y.; Yano, M.; Kawano, S.; Kizaki, N. JP 200521371, 2005.

[45] Onorato, C.; Emily, M.; Birthe, B.; Rama, V. US 9719071, 2007.

[46] Juan, M. S.; Busto, E.; Gotor, V.; Vicente, G. F. Org. Lett. 2013, 15, 3872 .

[47] Hyde, A. M.; Liu, Z. J.; Kosjek, B.; Tan, L. S.; Klapars, A.; Ashley, E. R.; Zhong, Y. L.; Alvizo, O.; Agard, N. J.; Liu, G. Q.; Gu, X. Y.; Yasuda, N.; Limanto, J.; Huffman, M. A.; Tschaen, D. M. Org. Lett. 2016, $18,5888$.

[48] Dimitris, K.; Ioulia, S. J. Org. Chem. 2010, 4, 8659.

[49] Danchet, S.; Bigot, C.; Buisson, D.; Azerad, R. Tetrahedron: Asymmetry 1997, 11, 1735.

[50] Kosjek, B.; Tellers, D. M.; Biba, M.; Farr, R.; Moore, J. Tetrahedron: Asymmetry 2006, 17, 2798.

[51] Musa, M. M.; Ziegelmann-Field, K. I.; Vieille, C.; Zeikus, J. G.; Phillips, R. S. J. Org. Chem. 2007, 72, 30.

[52] Feske, B. D.; Kaluzna, I. A.; Stewart, J. D. J. Org. Chem. 2005, 70, 9654.

[53] Marocco, C. P.; Davis, E. V.; Finnell, J. E.; Nguyen, P. H.; Mateer, S. C.; Ghiviriga, I.; Padgett, C. W.; Feske, B. D. Tetrahedron: Asymmetry. 2011, 22, 1784.

[54] Perrone, M. G.; Santandrea, E.; Scilimati, A.; Syldatk, C.; Tortorella, V.; Capitellic, F.; Bertolasi, V. Tetrahedron: Asymmetry 2004, 15 , 3511.

[55] Patal, R.; Banerjso, A.; Howell, J. M.; Mcnameo, C. G.; Brozozowski, D.; Mirfakhras, D.; Naaduri, V.; Thottathll, J. K.; Starka, L. J. Tetrahedron: Asymmetry 1993, 9, 2069.

[56] Kataoka, M.; Nakamura, Y.; Urano, N.; Ishige, T.; Shi, G.; Kita, S.; Sakamoto, K.; Shimizu, S. Lett. Appl. Microbiol. 2006, 43, 430.

[57] Kataoka, M.; Ishige, T.; Urano, N.; Nakamura, Y.; Sakuradani, E.; Fukui, S.; Kita, S.; Sakamoto, K.; Shimizu, S. Appl. Microbiol. Biotechnol. 2008, 80, 597.

[58] Urano, N.; Fukui, S.; Kumashiro, S.; Ishige, T.; Kita, S.; Sakamoto, K.; Kataoka, M.; Kita, S.; Shimizu, S. J. Biosci. Bioeng. 2011, 3, 266.

[59] Xu, F.; Chung, J. Y. L.; Moore, J. C.; Liu, Z. Q.; Yoshikawa, N.; Hoerrner, R. S.; Lee, J.; Royzen, M.; Cleator, E.; Gibson, A. G.; Dunn, R.; Maloney, K. M.; Alam, M.; Goodyear, A.; Lynch, J.; Yasuda, N.; Devine, P. N. Org. Lett. 2013, 6, 1342.

[60] Friest, J. A.; Maezato, Y.; Broussy, S.; Blum, P.; Berkowitz, D. B. J. Am. Chem. Soc. 2010, 132, 5930.

[61] Applegate, G. A.; Berkowitz, D. B. Adv. Synth. Catal. 2015, 357, 1619.

[62] Limanto, J.; Ashley, E. R.; Yin, J. J.; Beutner, G..; Grau, B. T.; Klapars, A. M.; Kim, M. M.; Klapars, A. P.; Liu, Z. J.; Strotman, H. R.; Truppo, M. D. Org. Lett. 2014, 16, 2716.

[63] Peng, Z. H.; Wong, J. H.; Hansen, E. C.; Puchlopek-Dermenci, A. L. A.; Clarke, H. J. Org. Lett. 2014, 16, 860. 